\title{
Optical feeder-link between ESA's optical ground station and Alphasat
}

Zoran Sodnik, Christoph Voland, Josep Perdigues, Edgar Fischer, Klaus Kudielka, et al.

Zoran Sodnik, Christoph Voland, Josep Perdigues, Edgar Fischer, Klaus Kudielka, Reinhard Czichy, "Optical feeder-link between ESA's optical ground station and Alphasat," Proc. SPIE 11852, International Conference on Space Optics - ICSO 2020, 1185218 (11 June 2021); doi: 10.1117/12.2599231

SPIE Event: International Conference on Space Optics - ICSO 2021, 2021, Online Only 


\section{International Conference on Space Optics-ICSO 2020}

Virtual Conference

30 March-2 April 2021

Edited by Bruno Cugny, Zoran Sodnik, and Nikos Karafolas
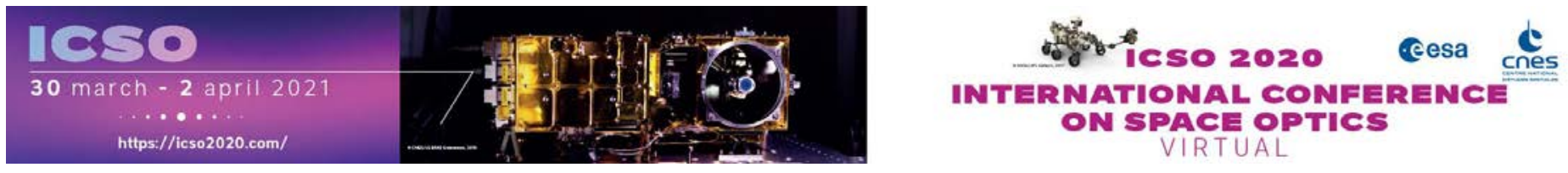

\section{Optical feeder-link between ESA's optical ground station and Alphasat}

\section{Cesa isoporecestings denes}




\title{
Optical feeder-link between ESA's optical ground station and Alphasat
}

\author{
Zoran Sodnik ${ }^{\mathrm{a}}$, Christoph Voland ${ }^{\mathrm{a}}$, Josep Perdigues ${ }^{\mathrm{a}}$, \\ Edgar Fischer ${ }^{\mathrm{b}}$, Klaus Kudielka ${ }^{\mathrm{b}}$, Reinhard Czichy ${ }^{\mathrm{b}}$ \\ aEuropean Space Agency, Keplerlaan 1, 2200AG Noordwijk, The Netherlands; \\ bynopta GmbH, Sonderstrasse 7, 9034 Eggersriet, Switzerland
}

\begin{abstract}
This paper describes the technical challenges and the experimental set-up for an optical feeder-link demonstration between ESA's optical ground station in Tenerife, Spain, and the Alphasat satellite in geostationary orbit $\left(25^{\circ}\right.$ East). In the absence of clouds, reliable optical feeder-uplinks require pre-distortion of the transmit beam to minimize the influence of the continuously changing atmospheric turbulence effect. Without beam pre-distortion, the quality of service (minimum impact of scintillations and outages) required for commercially viable feeder-links is hardly achievable.

Power efficient feeder-links require the wave-front distortions of an optical downlink beam from a satellite to be inversely applied onto the optical feeder-uplink beam to the satellite with certain adaptations due to the difference in the uplink and downlink paths. The underlying assumption is that pre-distortion and atmospheric turbulence cancel each other out and the main goal of the planned experiment is to determine to which extent this is true. The closed-loop bandwidth of pre-distortions has to be higher than the atmospheric turbulence fluctuations and optical beam reception and transmission should use the same telescope aperture. The former requires a fast controller and the later poses stringent requirement on the stray-light performance of the set-up such that the powerful transmit beam does not blind the receiver. Avoidance of receiver blinding is a particularly difficult task when doing feeder-uplink tests with the coherent laser communication terminal on board the Alphasat satellite as will be explained.
\end{abstract}

The paper will describe the design of the feeder-uplink system that will be installed in the OGS as well as initial measurements that have been performed to evaluate feasibility.

Keywords: Optical feeder-link, Sodium laser guide star, point ahead angle, wave-front pre-distortion, adaptive optics.

\section{INTRODUCTION}

Optical communication promises the possibility to feed (transmit) virtually unlimited amounts of data from optical ground stations (OGS) to future telecommunication satellites, without the need for radio frequency coordination. However, optical communication is facing two major problems when transmitting through the atmosphere; cloud coverage and atmospheric turbulence. While cloud coverage is unavoidable, it can be mitigated by feeder link channel diversity (placing multiple OGS in meteorologically uncorrelated locations - meaning $>400 \mathrm{~km}$ apart). However, reliable mitigation of atmospheric turbulence on the uplink has not been demonstrated yet. The reason being that the orbital motion of a spacecraft requires a small angular split between the transmitted and the received communication beams. This angular split, called point ahead angle (PAA), is tiny (about $18 \mu \mathrm{rad}$ for ground to GEO links), but it prevents deriving reliably wave-front distortions on the uplink from probing the wave-front distortions on the downlink. The result is that (depending on the strength of atmospheric turbulence) the uplink beam cannot be correctly pre-distorted (compensated) before passing atmospheric turbulence and the quality of service becomes unreliable. Depending on the strength of atmospheric turbulence a feeder-uplink beam may experience severe intensity fluctuations as well as outages when reaching the spacecraft. To minimize such effects it is necessary to implement a corresponding uplink beam shaping concept.

\section{OPTICAL FEEDER LINK ISSUES}

Optical communication links between space and ground are highly asymmetric because atmosphere turbulence occurs close to the optical ground station (OGS) and only over a few tens of kilometers. This asymmetry leaves only the OGS in a position to correct wave-front aberrations, because atmospheric turbulence occurs in its near-field. Near-field means 
that aberrations are present close to the OGS' aperture, which can be measured and inversely applied to the light received from the spacecraft. Such a system, consisting of a wave-front sensor (WFS) and a deformable mirror operating in closed loop is known as adaptive optics.

On the uplink, however, atmospheric turbulence occurs in the far-field of the spacecraft where no aberrations are present, because the transmit beam is distorted over the first tens of kilometer above the OGS and propagates undisturbed over the remainder of the link. Uplink beam distortions lead to beam spreading, de-pointing and interference (with extinction and amplification) effects that create a patchy speckle pattern in the far-field at the location of the spacecraft's optical communication terminal. The beam diameter when reaching the spacecraft is much larger than given by diffraction from the transmit aperture. As seen from the spacecraft, beam distortions take place at a point source in the far-field (at the OGS) causing the light received to be strongly fluctuating in irradiance, while the wave-front of the light (when received) is undistorted (perfectly flat) with no correction possible.

This leaves only the OGS with the capability to improve the quality on the uplink channel by inversely applying wavefront distortions to the uplink beam that have been measured on the downlink. The assumption being that atmospheric turbulence will undistort a correctly pre-distorted uplink beam [1]. The extent of validity of this assumption has so far not been tested in an uplink with a satellite. It is also understood that the solution proposed cannot be perfect, because wave-front sampling and beam pre-distortions never are. In addition, intensity aberrations (speckle) cannot be compensated on the uplink at all.

\subsection{Ground based experiments}

Optical feeder-links are difficult to test in ground-based experiments only. The unique scenario of a ground to space communication link, where atmospheric turbulence only occurs over the first tens of kilometers and with undisturbed free-space propagation over the majority of the link distance, is difficult to be reproduced on ground, especially when the point ahead angle (PAA) has to be taken into account. European companies and institutes have performed a number of ground-based experiments with very encouraging results, however, the proof of the results with a real feeder-uplink has yet still to come [2], [3], [4].

\subsection{Satellite based experiments}

The technology demonstration package \#1 (TDP\#1) on board the Alphasat satellite in Geostationary Earth Orbit (GEO, location is 25 degrees East) has been made available for feeder-link experiments by the German Space Agency (DLR). TDP\#1 consists of an optical communication terminal using coherent binary phase shift keying (BPSK) modulation at a wavelength of $1064 \mathrm{~nm}$ (with a few GHz frequency difference between Tx and Rx). This scheme is perfectly suited for inter-satellite communication, but may create Tx/Rx optical isolation problems in optical ground stations (OGS) if high uplink transmit powers are required. For optical communication uplinks with TDP\#1 the OGS so far used separate transmit and receive apertures (a so called bi-static arrangement). However, only wave-front distortions measured on the downlink can be used to predict the wave-front distortions on the uplink, which requires that Tx and Rx apertures are identical (a mono-static Tx/Rx arrangement).

The orbital motion of a spacecraft requires a small angular split between the transmitted and the received communication beams. This point ahead angle (PAA) is also dependent on the atmospheric turbulence conditions. Thus, it is difficult to derive the required uplink wave-front corrections from the downlink wave-front measurements only. The strength of atmospheric turbulence determines the isoplanatic angle, a measure of the angular cone within which wave-front distortions can be considered invariant. The point ahead angle (PAA) can be larger than the isoplanatic angle and the wave-front of the light received from TDP\#1 does not faithfully represent the conditions on the uplink.

A potential solution investigated by ESA is a technology used by astronomers to correct for atmospheric wave-front distortions, namely the creation of an artificial guide star (in the Sodium layer of the mesosphere in an altitude of about $90 \mathrm{~km}$ ). A powerful laser operating at $589 \mathrm{~nm}$ wavelength (the Sodium D-line) is projected into the sky that excites the Sodium atoms to form an artificial star. The Sodium guide star is placed at an angular distance away from the position of the telecommunication satellite that corresponds to the point ahead angle (PAA) and its wave-front is used to measure the wave-front distortions on the feeder-uplink.

Another well-known issue is caused by the simultaneous transmission and reception of the Sodium laser and artificial star light at the OGS. The light going up and the light coming down pass the same atmospheric turbulence layers and 
thereby cancel tip/tilt information, the largest contributor of wave-front errors. Thus, tip/tilt information needs to be obtained from the satellite downlink. As the isoplanatic angle for tip/tilt is larger than for higher order wave-front aberrations, determining tip/tilt from the satellite downlink should be feasible [5].

\section{OPTICAL FEEDER LINK DESIGN}

ESA is very actively pursuing optical feeder-link technologies for future terabit telecommunication satellites in the frame of the Optical Communication ScyLight program and has placed several technology demonstration activities to experimentally evaluate the extent to which atmospheric turbulence can be mitigated on a feeder-uplink channel and the technological effort that is required to do so. This is important in order to assess the cost/benefit ration of those concepts.

Optical feeder-link studies have shown that a terabit GEO satellite will need to be supported by $\sim 15$ widely separated $(>400 \mathrm{~km})$ optical ground stations (OGS) to avoid to be exposed to the same weather (correlation) patterns. The combined cloud-free line-of-sight (CFLOS) probability can then reach 99.9\%. The OGS with the highest CFLOS probability will operate most of the time, followed by a few more OGS with lower CFLOS probability; with the majority of OGS in the network being idle, only required to guarantee $99.9 \%$ availability of the complete system.

The switch over between different OGS is based on the individual feeder-uplink channel quality between OGS and satellite. The channel quality is affected (in order of severity, 1 being the worst) by:

1. Cloud coverage that leads to channel blockage, or unacceptable attenuation.

2. Atmospheric turbulence that exceeds the feeder-link system's ability to perform uplink pre-compensation.

3. Atmospheric turbulence that can only be pre-compensated based on wave-front measurements on a Sodium guide star.

4. Atmospheric turbulence that can be pre-compensated based on wave-front measurements of the downlink beam from the satellite.

It is obvious that conditions 1 and 2 immediately lead to a switch over from the satellite to another OGS in the network and the paper will, therefore, concentrate on conditions 3 and 4 only. The important parameter here is the statistics of the occurrence of condition 3 . If the probability for condition 3 turns out to be low compared to condition 4 , it may be possible to avoid the expensive installation and operation of Sodium guide star lasers in the OGS network.

The optical terminal on a terabit spacecraft in GEO will require a monitoring capability of the optical channels with all OGS in order to select the one with the best channel quality. The switch-over between any two OGS has to be performed in a make before break fashion. Each OGS may need to equalize the propagation delay between the data source/sink, its physical location and the link distance to the satellite with all other OGS such that the switch over from the satellite is performed without data gaps or data overlap.

\subsection{Feeder-uplink using the downlink beam for wave-front probing}

ESA developed the Cassegrain Adaptive Receiver Optics (CARO) for the OGS for reliable optical communication high capacity down-links from orbiting satellite [6]. CARO is operational at $1064 \mathrm{~nm}$ and $1550 \mathrm{~nm}$ wavelength bands and will also be used to obtain statistics on the channel quality and reliability that can be achieve on GEO data downlinks from TDP\#1 on board the AlphaSat satellite. Unfortunately, CARO cannot be used for pre-distortion of an optical transmit beam.

ESA is, therefore, developing adaptive pre-compensation systems for TDP\#1 data uplink. For efficient isolation (when transmitting and receiving from the same aperture) the transmit wavelength must be detuned from the receive wavelength to enable the use of band-pass filters. However, detuning means that TDP\#1 is not be able to receive data, because the local oscillator of its coherent receiver and tracking sensor only accepts a limited detuning range (a few tens of $\mathrm{GHz}$ ), only TDP\#1's acquisition sensor (it operates incoherently) is able to close the tracking loop. The acquisition sensor will, therefore, measure the dynamics of the uplink irradiance (scintillation) and will be used to evaluate the quality of the uplink wave-front correction by beam pre-distortion [7].

In an experimental uplink session with TDP\#1 in November 2019, the transmit beam from the OGS was deliberately detuned from $1064 \mathrm{~nm}$ until the acquisition sensor of TDP\#1 lost the uplink signal at $1081 \mathrm{~nm}$. This spectral acceptance range is a measure of the filter bandwidth in front of the acquisition sensor. Thus, a transmit wavelength of $1075 \mathrm{~nm}$ was 
selected for feeder-uplink experiments; a wavelength where standard commercial filters are available that provide sufficient blocking at $1064 \mathrm{~nm}$.

Figure 1 shows the principle experimental set-up that will used in the feeder-uplink system to be installed in ESA's OGS. A $20 \mathrm{~cm}$ diameter Tx/Rx aperture was selected, which is a sub-aperture of the 1 meter telescope in Coudé configuration. The $\mathrm{Tx} / \mathrm{Rx}$ wavelength separation of $11 \mathrm{~nm}$, together with orthogonal $\mathrm{Tx} / \mathrm{Rx}$ beam polarizations, enables efficient isolation from stray- and back reflected light. An issue was identified with the amplified spontaneous emission (ASE) of the fiber amplifier. While the seed laser is transmitting at $1075 \mathrm{~nm}$, the ASE spectrum of the fiber amplifier also covers (at much lower levels) the $1064 \mathrm{~nm}$ wavelength, which is strong enough to saturate (blind) the wave-front sensor. A $1075 \mathrm{~nm}$ band-pass is, therefore, placed at the $1075 \mathrm{~nm}$ Tx laser output. Uplink-tests with the TDP\#1 optical communication terminal are scheduled for end 2021.
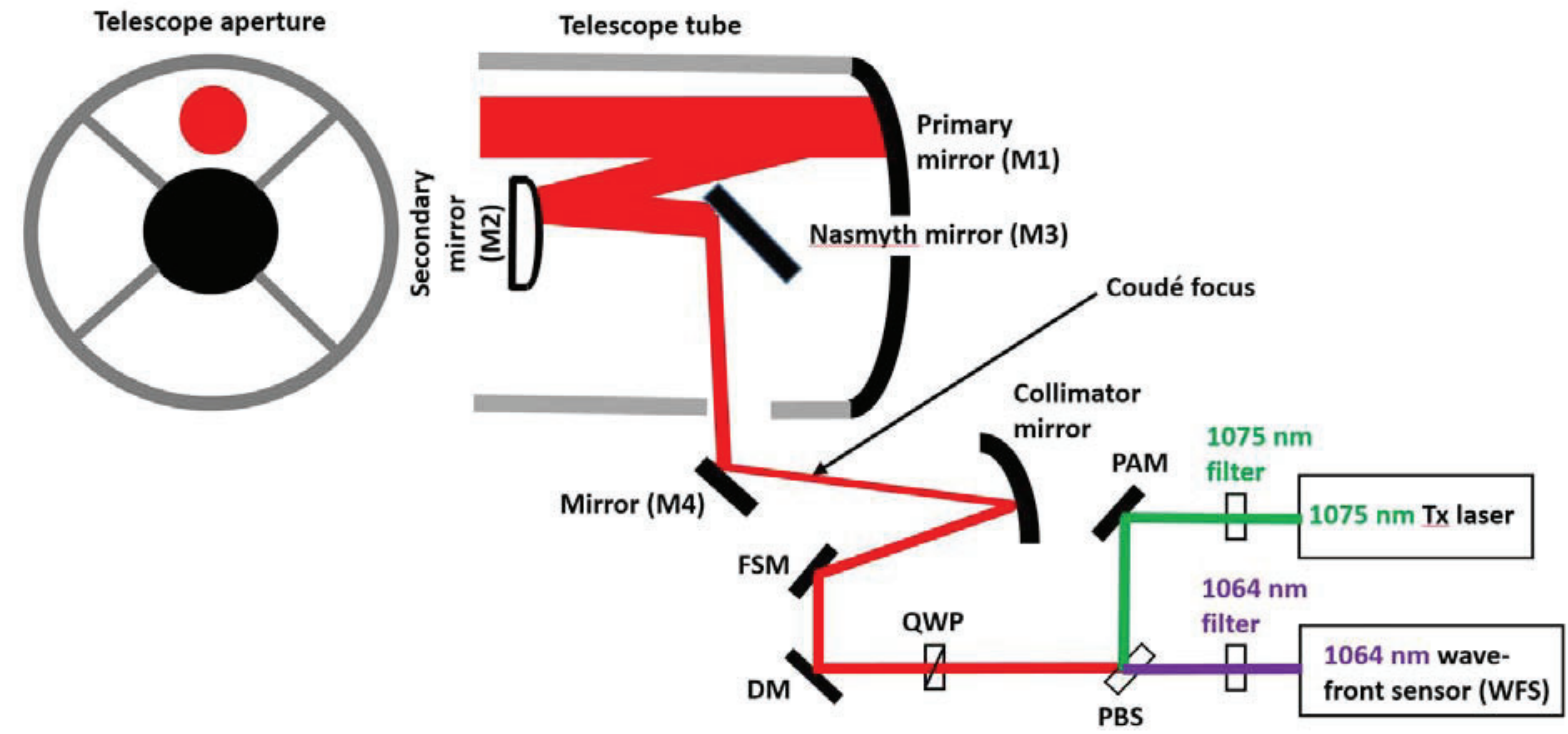

Figure 1: Schematic drawing of the feeder-uplink test arrangement without a Sodium guide star to be implemented in the OGS. Folding mirrors, the Tx/Rx alignment scheme, pupil and other imaging optics are omitted. The acronyms are: FSM (Fine Steering Mirror), DM (Deformable Mirror), QWP (Quarter Wave Plate), PAM (Point Ahead Mirror) and PBS (Polarizing Beam Splitter).

\subsection{Feeder-uplink using a Sodium laser guide star for wave-front probing}

In addition to the uplink experiment reported in the previous chapter, ESA is preparing an invitation to tender (ITT) for the development of an optical communication system that uses a Sodium laser to generate a guide star for wave-front probing on the uplink [8], [9], [10]. The system will also be installed in the OGS, transmitting the Sodium laser beam out of the full 1 meter telescope aperture. Only in case of isolation problems the Sodium laser beam will be transmitted from a $20 \mathrm{~cm}$ guider telescope, which is attached and co-aligned with the main telescope.

Figure 2 depicts the feeder-uplink set-up. It is only schematic, omitting (pupil) imaging optics, acquisition camera, transmit/receive laser beam alignment components as well as means to avoid alignment drifts during operations. Transmission and reception of the feeder link communication beams and reception of the light from the Sodium guide star are performed via a $200 \mathrm{~mm}$ diameter sub-aperture of the 1 meter OGS telescope. Tip/tilt correction is performed by a fine steering mirror (FSM) and measured by a "1064 nm tracking sensor (TS)" based on the communication beam from 
the GEO satellite, while higher-order feeder uplink pre-distortions are performed with a deformable mirror (DM) and measured by a "589 nm wave-front sensor (WFS)" based on wave-front measurements on the Sodium guide star light.

The WFS is located behind an ultra-narrow "Sodium line $589 \mathrm{~nm}$ filter" (to remove the daylight blue-sky brightness), a Dichroic Beam Splitter (DBS, that separates the $1075 \mathrm{~nm}$ transmit beam from the light from the Sodium guide star at 589 nm), a Point Ahead Mirror (PAM), a Deformable Mirror (DM) and a beam splitter (BS) that reflects most of the light and transmits a small amount that is sufficient for tip/tilt beam probing via the $1064 \mathrm{~nm}$ tracking sensor (TS).

Feeder-link transmitter/receiver co-alignment is performed by switching the transmit beam to minimum power and by moving a motorized retro-reflector between FSM and a Mirror with eccentric Hole (MwH). The point ahead mirror (PAM) is adjusted such that zero tilt is measured by the $1064 \mathrm{~nm}$ tracking sensor (TS). With the $1075 \mathrm{~nm}$ laser beam retro-reflected towards the $1064 \mathrm{~nm}$ tracking sensor (TS), the tiny transmission of the $1064 \mathrm{~nm}$ filter at $1075 \mathrm{~nm}$ is sufficient to perform this alignment. The transmit laser is then switched off, the Sodium laser on (to minimum power) and the tip/tilt mirror (TTM) is set to the same zero tilt position on the $1064 \mathrm{~nm}$ tracking sensor (TS) as measured before. Again it is assumed that when directly retro-reflected the transmission of the $1064 \mathrm{~nm}$ filter at $589 \mathrm{~nm}$ is sufficient to perform the alignment. The DM is adjusted for minimum wave-front error (WFE) and zero tilt as measured by the WFS and PAM is set to the required point ahead angle (PAA) for the GEO satellite. The TTM is adjusted such that the WFS measures the same zero tilt as before and the retro-reflector is removed. With the telescope pointed towards the satellite and sufficient Sodium laser power the WFS should measure wave front distortions of the light from the Sodium guide star. After this, the feeder uplink system is ready for operation upon acquisition of the light from the GEO spacecraft.

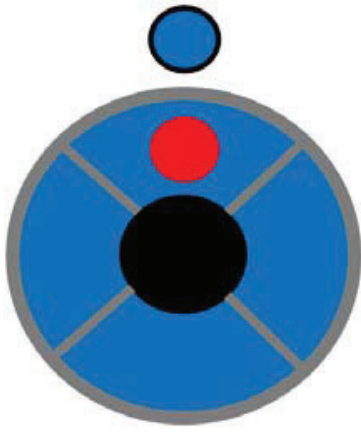

Telescope aperture

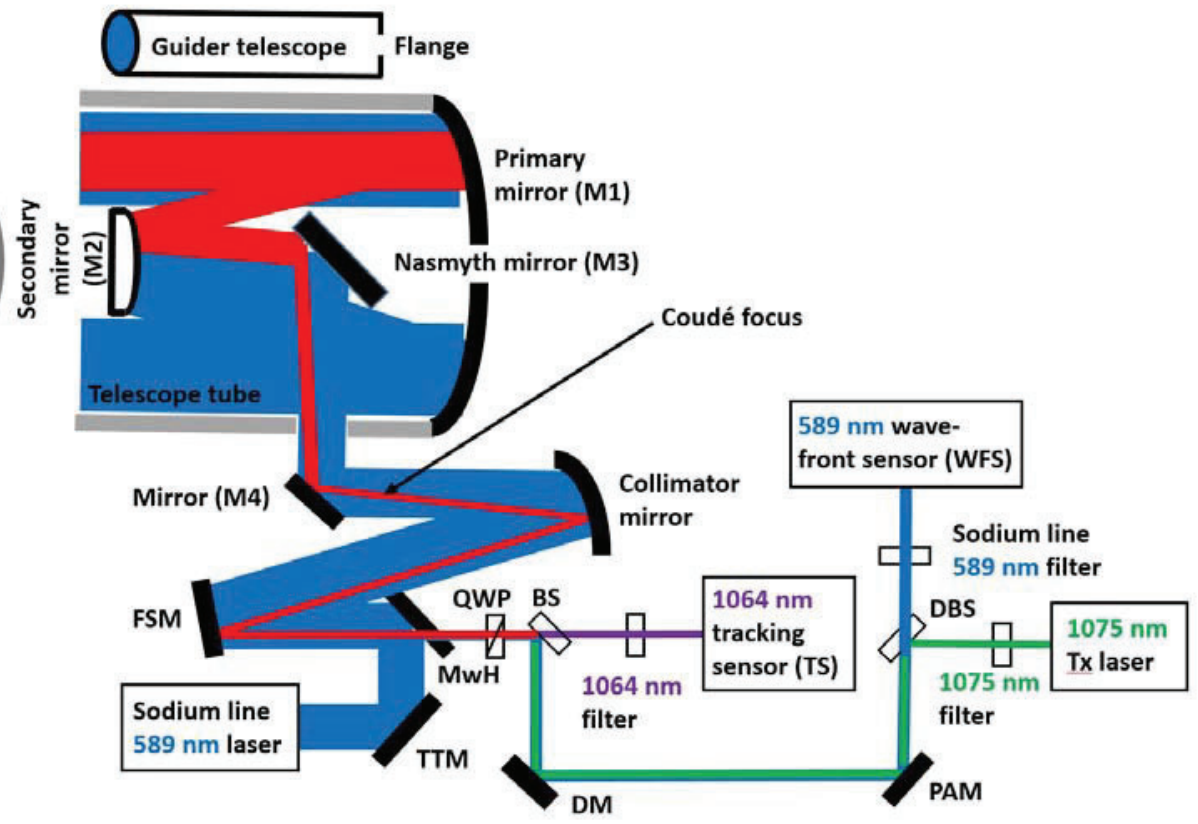

Figure 2: Schematic drawing of the feeder-uplink test arrangement with a Sodium guide star that will be implemented in the OGS. Folding mirrors, the Tx/Rx alignment scheme, pupil and other imaging optics are omitted. The acronyms are: FSM (Fine steering mirror), DM (deformable mirror), QWP (quarter wave plate), PAM (Point ahead mirror) DBS (Dichroic Beam Splitter), TTM (Tip/Tilt Mirror), MwH (Mirror with Hole) and BS (Beam Splitter). 


\section{CONCLUSIONS}

This paper listed the most important issues (known at the time of writing) that will be taken into account for the development of a feeder-uplink system to be installed in ESA's optical ground station (OGS) in Tenerife, Spain. The experimental set-ups presented are initial proposals and improved solution may well be identified during experiments.

Feeder-links based on optical technologies may open a door to the vast bandwidth resources promised by optical wavelengths that satellite telecom operators will require in the not too distant future. There is a great chance that optical technologies can deliver the promise and master atmospheric turbulence, but this has to be proven first. Feasibility will also depend on the technological effort required, the cost, and on the ability to master laser safety aspects involved.

ESA and its Optical Communication ScyLight funding scheme for telecommunication applications are committed to develop experimental evidence within the coming two years.

\section{ACKNOWLEDGEMENTS}

The authors are grateful for the support of Rolf Meyer and Björn Gütlich from the German Space Agency (DLR) and for valuable discussions with Domenico Bonaccini Calia from the European Southern Observatory (ESO), David Alaluf from ESA, Frank Lison from the German company Toptica and Nicolas Vedrenne from the French company ONERA.

\section{REFERENCES}

[1] Kudielka K., Fischer E. and Dreischer T., "Numerical prediction and experimental validation of irradiance fluctuations in a pre-compensated optical feeder link", Proc. SPIE 11180, (2010).

[2] Védrenne N. et al., "First experimental demonstration of adaptive optics pre-compensation for GEO feeder links in a relevant environment", 2019 IEEE International Conference on Space Optical Systems and Applications (ICSOS)

[3] Montmerle Bonnefois A. et al., "Adaptive Optics pre-compensation for GEO feeder links: the FEEDELIO experiment", Proc. SPIE 11180, (2010).

[4] Saathof R. et al., "Adaptive Optics pre-correction for Optical Feeder Links - breadboard performance", Proc. SPIE 11180, (2019).

[5] Alaluf D., Perdigues Armengol J. and Sodnik Z., "How effective is tip-tilt pre-compensation for optical uplinks based on the received downlink optical signal?", Proc. SPIE 11180, (2019).

[6] Fischer E. et al., "ESA Optical Ground Station Upgrade with Adaptive Optics for High Data Rate Satellite-toGround Links - Test Results", 2019 IEEE International Conference on Space Optical Systems and Applications (ICSOS)

[7] Camboulives A-R., Velluet M-T., Poulenard S., Saint-Antonin L. and Michau V., "Optical Ground Station optimization for future optical geostationary satellite feeder uplinks", Proc. SPIE 10096, (2017).

[8] D’Orgeville C. et al., "A Sodium Laser Guide Star Facility for the ANU/EOS Space Debris Tracking Adaptive Optics Demonstrator", Proc. SPIE 9148, (2014).

[9] Zuraski S. and Fiorino S., "World Wide Study of the Performance of a Sodium Guidestar", Proc. SPIE 9224, (2014).

[10] Mata Calvo R. et al., "Laser Guide Stars for Optical Free-Space Communications”, Proc. SPIE 10096, (2017). 